\title{
Damage-Induced Neuronal Endopeptidase Is Critical for Presynaptic Formation of Neuromuscular Junctions
}

\author{
Kenichi Nagata, ${ }^{1}$ Sumiko Kiryu-Seo, ${ }^{1}$ Mitsuyo Maeda, ${ }^{1}$ Kayo Yoshida, ${ }^{2}$ Takashi Morita, ${ }^{2}$ and Hiroshi Kiyama ${ }^{1}$ \\ Departments of ${ }^{1}$ Anatomy and Neurobiology, and ${ }^{2}$ Molecular Genetics, Osaka City University, Graduate School of Medicine, Osaka 545-8585, Japan
}

\begin{abstract}
Damage-induced neuronal endopeptidase (DINE) is a metalloprotease belonging to the neprilysin family. Expression of DINE mRNA is observed predominantly in subsets of neurons in the CNS and peripheral nervous system during embryonic development, as well as after axonal injury. However, the physiological function of DINE and its substrate remain unknown. We generated DINE-deficient mice to examine the physiological role of DINE. Shortly after birth, these mice died of respiratory failure resulting from a dysfunction of the diaphragm, which showed severe atrophy. As DINE was abundantly expressed in motor neurons and there was atrophy of the diaphragm, we analyzed the interaction between motor nerves and skeletal muscles in the DINE-deficient mice. Although there were no obvious deficiencies in numbers of motor neurons in the spinal cord or in the nerve trajectories from the spinal cord to the skeletal muscle in DINE-deficient mice, detailed histochemical analysis demonstrated a significant decrease of nerve terminal arborization in the diaphragm from embryonic day 12.5. In accordance with the decrease of final branching, the diaphragms from DINE-deficient mice exhibited only a few neuromuscular junctions. Similar changes in nerve terminal morphology were also apparent in other skeletal muscles, including the latissimus dorsi and the intercostal muscles. These data suggest that DINE is a crucial molecule in distal axonal arborization into muscle to establish neuromuscular junctions.
\end{abstract}

\section{Introduction}

Damage-induced neuronal endopeptidase (DINE) was originally identified as a nerve injury-associated gene by differential display PCR (Kiryu et al., 1995; Tanabe et al., 1999; Kiryu-Seo et al., 2000). DINE is a membrane-bound metalloprotease. Its expression is markedly increased in response to nerve injury and DINE shares high homology with endothelin-converting enzyme (ECE) and neprilysin (NEP) (Devault et al., 1987; Xu et al., 1994). The human ortholog of DINE was separately identified as an ECE-1 homologous gene using expressed-sequence tag (EST) analysis where the ECE-1 sequence was compared with a collection of EST cDNA clones obtained from a human tumorous neuroganglion tissue library. This gene was initially termed XCE (Valdenaire et al., 1999), but was later registered as endothelin-converting enzyme-like 1 (ECEL1) on the basis of its strong similarity to ECE-1 (Valdenaire and Schweizer, 2000). Despite the structural similarities between DINE, ECE, and NEP (Turner et al., 2001), DINE does not cleave ECE and NEP substrates such as big endothelins and amyloid $\beta$ (Kiryu-Seo et al., 2000; Shirotani et al., 2001), and the physiological function of DINE (ECEL1) and its substrate remain unknown.

DINE exhibits two expression profiles distinct from other members such as ECE and NEP. First, DINE is expressed specif-

Received Sept. 10, 2009; revised March 12, 2010; accepted April 6, 2010.

This work was supported by grants from Ministry of Health, Labour and Welfare of Japan; Ministry of Education, Culture, Sports, Science, and Technology of Japan; and Japan Science and Technology Agency. We thank R. Miyabe for secretarial assistance. We are grateful to C. Kadono for her excellent technical assistance.

Correspondence should be addressed to Dr. Hiroshi Kiyama, Department of Anatomy and Neurobiology, Osaka City University, Graduate School of Medicine, 1-4-3 Asahimachi, Abeno-ku, Osaka 545-8585, Japan. E-mail: kiyama@med.osaka-cu.ac.jp.

DOI:10.1523/JNEUROSCI.4521-09.2010

Copyright $\odot 2010$ the authors $\quad 0270-6474 / 10 / 306954-09 \$ 15.00 / 0$ ically in neurons of the CNS and peripheral nervous system (PNS) from early developmental stages (Nagata et al., 2006) and is expressed strongly in some groups of neurons originating from the basal plate, including the hypothalamus and cranial motor neurons, and in peripheral ganglia derived from the neural crest. Such expression patterns are particularly high during embryonic development, suggesting that DINE plays an important role in neuronal development. The other characteristic DINE expression profile is its marked induction in response to various types of neuronal injury (Kiryu-Seo et al., 2008). For instance, peripheral and optic nerve transections induce DINE mRNA expression in nerve-injured neurons (Kiryu-Seo et al., 2000; Kato et al., 2002). Similarly, in cerebral ischemia, late-onset and prolonged DINE mRNA expression was observed in cortical and thalamic neurons that were projecting their axons to the infarct region (Ohba et al., 2004). Thus, DINE expression seems highly associated with nerve injury in both the CNS and PNS.

ECEL1-deficient mice have been previously developed, although the mice died immediately after birth due to respiratory failure and atelectasis (Schweizer et al., 1999). Nevertheless, obvious histological abnormalities were not precisely examined. In the present study, to determine the exact cause of respiratory failure, we performed a detailed analysis of the precise abnormalities of peripheral nerve structure during embryonic periods by producing an alternative line of DINE-deficient mice. Although these DINE-deficient mice also died immediately after birth due to respiratory failure, we were able to identify failure of final branching of motor nerves to the endplate, resulting in the poor formation of neuromuscular junctions (NMJ). These data suggest that DINE is crucial for distal axonal arborization into muscle to establish NMJ. 


\section{Materials and Methods}

Animals. A DINE-targeting construct was designed to insert a loxP sequence into intron 12 and another loxP into intron 15 with a neomycinresistant gene as a selective marker (see Fig. $1 \mathrm{~A}$ ). The vector was constructed from DNA of BAC clone RP23-416O18 by using Tyramide Signal Amplification-Direct (Red) system (Copeland et al., 2001). The 5' $\operatorname{arm}(4.6 \mathrm{~kb})$ of the vector started from the sequence ATCCTGGCGGCCATGCTGGC in exon 2 and terminated at CAAAGGCATG in intron 12, where the first loxP sequence was introduced. The central arm $(1.4 \mathrm{~kb})$ of the vector extended from the rest of intron 12 of GTCACAGGCCAGTCC to intron 15 of AGAGTAGAAA. The $3^{\prime}$ arm started from the phosphoglycerate kinase promoter-driven neomycin-resistant gene and the second loxP sequence, which were followed by GCTTGACAGGCAGGGAAT in intron 15 through to the $3^{\prime}$ untranslated region of the DINE gene CTGGTGTGCACTGCTAGCAA $(6.8 \mathrm{~kb})$. The targeting vector was linearized by a PacI site at the $5^{\prime}$ end and was used to transfect RI embryonic stem (ES) cells. Successful targeting of the DINE gene was achieved in G418 resistant clones, as judged by Southern blot analysis. The targeted ES clones were used to produce chimeras by an aggregation method. One female chimera and one male chimera were born, which were derived $100 \%$ from ES cells as determined from their coat color (agouti). These animals transmitted the targeted allele to half of their progeny by mating with C57BL/6 mice. However, crosses between heterozygotes produced no homozygous offspring by PCR.

DINE-deficient animals were backcrossed for at least six generations to C57BL/6J mice. The homozygous DINE-deficient mice were obtained by intercrossing the heterozygous parents. Noon on the day of the detection of the vaginal plug was considered embryonic day 0.5 (E0.5). Tissue lysates from mice were used for PCR analysis with allele-specific primers: DINE-WILD, 5'-CCCCACCAGCCCCGGTTATGTTATCC-3'; DINEPOL2, 5' -CGCATCGCCTTCTATCGCCTTCTTGACGAG-3'; and DINEup, 5'-GCTGGGGGACAGGTGGGAGCTGATGA-3'. The reaction was cycled 30 times $\left(30 \mathrm{~s} 94^{\circ} \mathrm{C}, 30 \mathrm{~s} 60^{\circ} \mathrm{C}\right.$, and $30 \mathrm{~s} 72^{\circ} \mathrm{C}$ ), which amplified a 280-bp fragment in wild-type animals, a 360-bp fragment in knock-out mice, and both 280-bp and 360-bp fragments in heterozygous animals. All experimental procedures were conducted in accordance with standard guidelines for animal experiments of the Graduate School of Medicine, Osaka City University (Osaka, Japan).

Reverse transcriptase-PCR. Total RNA was extracted from spinal cord of E18.5 mice or diaphragms of E14.5 mice, and was converted to cDNA with Superscript reverse transcriptase III (Invitrogen) and nucleotide oligo-dT18. Aliquots from the reverse transcriptase (RT) reaction were used for PCR amplification using primer pairs ubiquitously expressing GAPDH as a control. The DINE-specific primer sequences for PCR were as follows: 5'-CAGCAGCTGGTGGAAGATATCAAG-3' (P1) and 5'GAATTCCTCAAACTGGGACACGCTACC-3' (P2) (Fig. 1A). These primers were deduced from mouse sequence located at $5^{\prime}$ (Fig. $1 A$, eighth exon) and 3' (Fig. 1 A, 18th exon). Primers used in PCR analysis for the detection of GAPDH were as follows: $5^{\prime}$-AGGTCATCCATGACAACTTTGG- $3^{\prime}$ and 5' ${ }^{\prime}$-CAGGTTTCTCCAGGCGGCATGT- ${ }^{\prime}$. For the semiquantitative RT-PCR analysis, cDNA was amplified using the following primers: MuSK-interacting cytoplasmic adaptor-like protein (Dok-7; 254-bp fragment), 5'-CTCCGCAGTGGAAGAGTAGGT-3' and $5^{\prime}$-CCAGGCACACATGGCCTCATGGCT-3'; low-density lipoprotein receptor-related protein 4 (LRP4; 300-bp fragment), 5'-AGTGCTGCCCACATTCTCTTC- $3^{\prime}$ and 5'-GCTCTGTGCTGACAAGGAATC3'; muscle-specific receptor tyrosine kinase (MuSK; 537-bp fragment), 5'-CAACATTCCCGTCAATAACGTC-3' and 5'-GTTTCACAATGTTGGGGTTGTC-3'; AChR $\alpha$ (385-bp fragment), 5'-CACTTTCCCTTCGATGAGCAGA-3' and 5'-CCACAATGACCAGAAGGAACAC-3'. The reaction products were electrophoretically separated on $1 \%$ agarose gel and visualized by staining with ethidium bromide.

Preparation of recombinant DINE and generation of anti-DINE antibody. To generate recombinant DINE-His fusion proteins, partial sequences for amino acids 143-465 of DINE (see Fig. 2 A) were amplified from full-length mouse DINE cDNA and subcloned into pET-30a (+) (Novagen). PCR amplification was performed using the upstream primer $5^{\prime}$-CCATATGGACAAGCTCACCTAT-3' and the downstream primer 5'-GCTCGAGGGCAGCTGAGAAGTG-3'. The amplified DINE gene encoding 323 aa residues was subcloned into pET-30a (+) (Novagen) between the NdeI and XhoI sites. BL21 (DE3) bacteria transformed with those vectors were stimulated with $1 \mathrm{~mm}$ isopropyl-Dthiogalactopyranoside for $3 \mathrm{~h}$ at $37^{\circ} \mathrm{C}$, harvested by brief centrifugation, and lysed in PBS containing 1\% Triton X-100 for $30 \mathrm{~min}$ on ice. The product was pelleted by centrifugation, incubated with binding buffer (20 mM NaH $\mathrm{m}_{2} \mathrm{PO}_{4} .2 \mathrm{H}_{2} \mathrm{O}, 0.5 \mathrm{M} \mathrm{NaCl}, 10 \mathrm{~mm}$ imidazole, and $8 \mathrm{M}$ urea, $\mathrm{pH}$ 7.5) for $3 \mathrm{~h}$ at $4^{\circ} \mathrm{C}$, and then centrifugation at 10,000 rpm for $20 \mathrm{~min}$ at $4^{\circ} \mathrm{C}$. The supernatant was collected and centrifuged again at 10,000 rpm for $20 \mathrm{~min}$. The supernatant was filtered and applied to a HisTrap HP column using an AKTAprime system (both from GE Healthcare BioSciences) according to the manufacturer's instructions. Briefly, the column was washed with five volumes of binding buffer and the proteins were eluted with a stepwise gradient $(0-100 \%)$ of elution buffer $(20 \mathrm{~mm}$ $\mathrm{NaH}_{2} \mathrm{PO}_{4} .2 \mathrm{H}_{2} \mathrm{O}, 0.5 \mathrm{M} \mathrm{NaCl}, 500 \mathrm{~mm}$ imidazole, and $8 \mathrm{M}$ urea, $\mathrm{pH} 7.5$ ). The purified proteins were checked by SDS-PAGE followed by Coomassie Brilliant Blue R-250 staining and stored at $-80^{\circ} \mathrm{C}$ until use. A rabbit polyclonal anti-DINE antiserum was raised against the recombinant DINE (143-465aa) described above (Fig. $2 A$ ) and the antiserum obtained was concentrated by ammonium sulfate precipitation. The anti-DINE antiserum was further purified by chromatography through an affinity column in which the recombinant DINE was coupled to Hi-Trap NHSactivated HP (GE Healthcare Bio-Sciences) according to the manufacturer's instructions.

Western blotting. E18.5 mice spinal cord was homogenized in $45 \mathrm{~mm}$ Tris and $8 \mathrm{~m}$ urea (lysis buffer). The homogenate was solubilized with lysis buffer containing 2\% 3-[(3-cholamidopropyl)dimethylammonio]1-propanesulfonate for $30 \mathrm{~min}$ on ice and centrifuged at 10,000 rpm for $15 \mathrm{~min}$. The supernatant was used for further analysis. Immunoblot analysis was performed using the DINE antibody (1:300) and horseradish peroxidase-conjugated anti-rabbit IgG secondary antibodies, as recommended by the manufacturer (GE Healthcare Bio-Sciences).

Histological analysis and immunohistochemistry. Embryos at various stages and mice at $0 \mathrm{~d}$ of age (P0) were collected, and all animals and organs were fixed in phosphate-buffered $4 \%$ paraformaldehyde (PFA) overnight, then immersed in $0.1 \mathrm{~m}$ phosphate buffer containing $30 \%$ sucrose for an additional day. P0 mice were embedded in OCT compound (TissueTek; Sakura Finetek), and serial $16 \mu \mathrm{m}$ sections were cut on a cryostat and stained with hematoxylin and eosin (H\&E). Embryos were embedded in OCT compound, and serial 10 or $16 \mu \mathrm{m}$ sections were cut on a cryostat and stained with H\&E or used for immunohistochemistry. Sections of the embryonic diaphragm were counted with the experimenter blinded to the treatment groups, and the mean value of these counts was used as an individual data point for statistical analysis by Student's $t$ test $(p<0.05)$. Wild-type mice $(n=4)$ were analyzed with heterozygous mice $(n=4)$ and DINE-deficient mice $(n=4)$. Immunohistochemistry was performed essentially as described previously (Kopp et al., 1997), with minor alternations. The sections were rinsed three times in PBS, permeabilized by immersion in absolute $\mathrm{MeOH}$ for 6 min at $-30^{\circ} \mathrm{C}$ (except for the use of anti-choline acetyltransferase and $\alpha$-bungarotoxin), rinsed in PBS for $30 \mathrm{~min}$, and blocked for $30 \mathrm{~min}$ in a solution (diluent) consisting of $0.3 \%$ Triton X-100, $0.2 \%$ bovine serum albumin (BSA), and $0.1 \%$ sodium azide in PBS. The sections were then incubated with rabbit anti-DINE primary antibody (1:300), monoclonal anti-neurofilament antibody (2H3; Developmental Studies Hybridoma Bank), and goat anti-choline acetyltransferase (ChAT) primary antibody (1:300; Millipore) in the diluent at room temperature overnight on a shaker plate. Tissue was rinsed in diluent for $30 \mathrm{~min}$, the appropriate secondary antibodies and/or $\alpha$-bungarotoxin (BTX) conjugated with Alexa Fluor 594 (1:300; Invitrogen) were applied for $1 \mathrm{~h}$, and then tissues were rinsed three times in PBS. The sections were visualized by fluorescent microscopy (AX70; Olympus).

For quantification of motor neurons, the location of C3-C5 at E18.5 was determined morphologically based on the surrounding tissue. ChAT-immunopositive motor neurons were counted independently on the right and left hemiventral columns in every fourth section. Hemiventral columns from each animal were counted with the experimenter blinded, and the mean value of those counts was used as an individual 
data point for statistical analysis by Student's $t$ test $(p<0.05)$. DINEdeficient mice $(n=3)$ were analyzed with their wild-type littermates $(n=3)$.

Whole-mount immunohistochemistry. For diaphragm staining, wholemount immunohistochemistry was performed essentially as described previously (Lin et al., 2005). Briefly, diaphragm muscles were fixed in $2 \%$ PFA in $0.1 \mathrm{M}$ phosphate buffer, $\mathrm{pH} 7.4$, overnight at $4^{\circ} \mathrm{C}$, rinsed briefly with PBS, pH 7.4, incubated in $0.1 \mathrm{M}$ glycine in PBS for $1 \mathrm{~h}$, rinsed briefly with PBS, and then washed with $0.5 \%$ Triton X-100 in PBS. The muscles were blocked in dilution buffer $(500 \mathrm{~mm} \mathrm{NaCl}, 0.01 \mathrm{~m}$ phosphate buffer, $3 \%$ BSA, $5 \%$ goat serum, and $0.01 \%$ thimerosal) overnight at $4^{\circ} \mathrm{C}$, and then incubated with antibodies against neurofilament-M (1:1000; Millipore) or S100 (1:400; Dako) in dilution buffer overnight at $4^{\circ} \mathrm{C}$. After washing three times for $1 \mathrm{~h}$ each in $0.5 \%$ Triton X-100 in PBS, the muscles were incubated with anti-rabbit IgG conjugated with Alexa Fluor 488 (1:500; Invitrogen) and BTX conjugated with Alexa Fluor 594 (1:300; Invitrogen) overnight at $4^{\circ} \mathrm{C}$. The muscles were then washed three times for $1 \mathrm{~h}$ each with $0.5 \%$ Triton X-100 in PBS and twice with PBS, and were visualized by fluorescent microscopy. Stained diaphragms were photographed and the branches on the phrenic nerve were counted. E12.5 DINE-deficient mice $(n=3)$ were analyzed with wild-type littermates $(n=3)$.

Embryos were stained as previously described (Qiu et al., 1997). Briefly, embryos were collected in PBS and fixed in methanol:DMSO (4:1) overnight at $4^{\circ} \mathrm{C}$. The embryos were then bleached in methanol: DMSO:30\% $\mathrm{H}_{2} \mathrm{O}_{2}$ (4:1:1) for $4-5 \mathrm{~h}$ at room temperature, and rehydrated for 30 min each through 50\% and 15\% methanol into a final PBS wash. Embryos were incubated twice in PBSMT (2\% instant skim milk powder, $0.1 \%$ Triton $\mathrm{X}-100$ in $\mathrm{PBS}$ ) for $1 \mathrm{~h}$ at room temperature, and then incubated with a monoclonal antibody $(2 \mathrm{H} 3)$ recognizing a $165 \mathrm{kDa}$ neurofilament protein in PBSMT at $4^{\circ} \mathrm{C}$ overnight. Embryos were washed in PBSMT twice at $4^{\circ} \mathrm{C}$ and three times at room temperature for $1 \mathrm{~h}$ each, followed by an overnight incubation with peroxidaseconjugated goat anti-mouse IgG diluted in PBSMT at $4^{\circ} \mathrm{C}$. The washes were the same as for after primary antibody, except with an additional 20 min wash in PBST $(0.2 \%$ BSA, $0.1 \%$ Triton X-100 in PBS) at room temperature. For the color reaction, embryos were incubated with 0.3 $\mathrm{mg} / \mathrm{ml}$ diaminobenzidine tetrahydrochloride (Sigma-Aldrich) in PBST for 30-60 $\mathrm{min}$ at room temperature, and then visualized by addition of $0.0003 \% \mathrm{H}_{2} \mathrm{O}_{2}$ and incubation at room temperature. Embryos were rinsed in PBST to stop the reaction, dehydrated through a methanol series $(30 \%, 50 \%, 80 \%$, and $100 \%$ for $30-60 \mathrm{~min}$ each), and cleared in benzyl alcohol:benzyl benzoate (1:2). Embryos were photographed by fluorescent microscopy.

Electron microscopy. For ultrastructural study, embryonic muscles were fixed in $2 \%$ glutaraldehyde and $2 \%$ paraformaldehyde in $0.1 \mathrm{M}$ phosphate buffer, washed, further fixed in $1 \%$ OsO4, dehydrated, and embedded in resin. Thin sections were stained with uranyl acetate and lead citrate.

Retrograde tracing experiments. To trace the projection of sensory afferents, mouse embryos were immersion fixed in $4 \%$ paraformaldehyde in PBS and stored in fixative until the application of axon tracer. Sensory projections were labeled with a saturated solution of the lipid-soluble fluorescent dye 1,1'-dioctadecyl-3,3,3',3-tetramethylindocarbocyanine perchlorate (DiI; Invitrogen) in ethanol. DiI was injected into DRGs using glass capillaries. The preparations were then incubated in $4 \%$ paraformaldehyde buffer at $37^{\circ} \mathrm{C}$ for $2-4 \mathrm{~d}$ before detection of DiI.

\section{Results}

\section{DINE-deficient mice are lethal after birth due to} respiratory dysfunction

We initially designed conditional DINE knock-out mice (KO) where two loxP sites were inserted into introns 12 and 15 to remove the active site of DINE protease by Cre recombinase (Fig. $1 A)$. As the DINE gene was modified to include two loxP and a neomycin-resistant gene in intron 12 and 15, we assumed that the DINE gene should function normally until the exons 13,14, and 15 were removed by Cre recombinase. However, our finding of
A

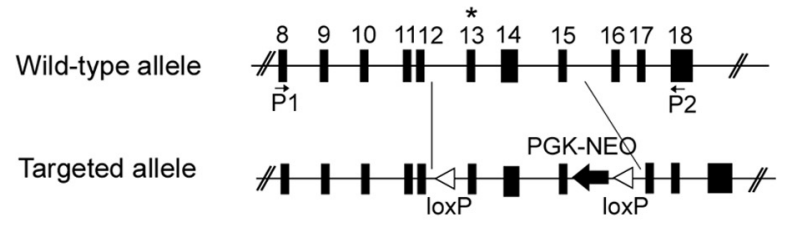

B

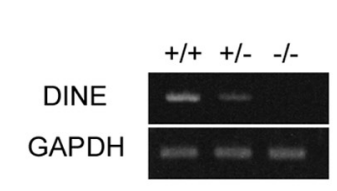

D
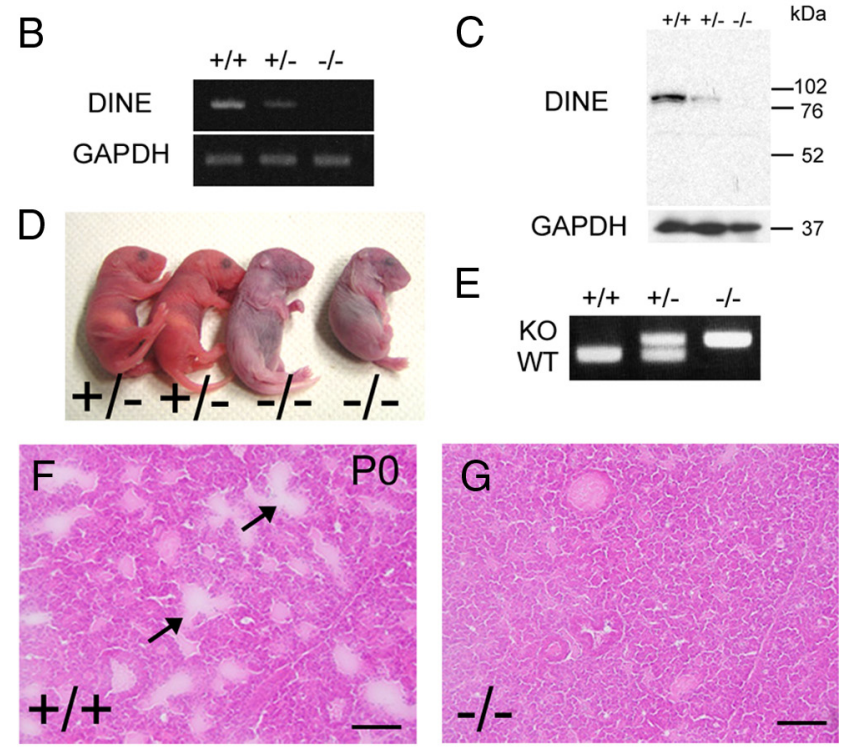

$\mathrm{H}$
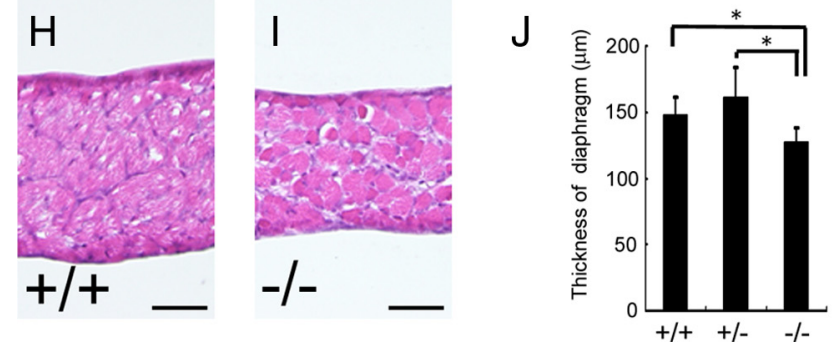

Figure 1. DINE-deficient mice die of respiratory failure immediately after birth. $A$, Schematic representation of a segment of the wild-type mouse DINE allele and the targeted DINE allele. Solid black boxes represent the last 11 exons and the marked 13th exon (*) includes the zincbinding motif of DINE. The neomycin resistance gene (PGK-NEO), two loxP sequences (loxP), and RT-PCR primers (arrows; $\mathrm{P} 1, \mathrm{P2}$ ) used are shown. $B$, RT-PCR analysis using total RNAs from the embryonic spinal cords of wild-type $(+/+)$, heterozygous $(+/-)$, and DINE-deficient $(-/-)$ mice. DINE-specific primers (P1 and P2) were created from sequence of the 8 th and 18 th exon as shown in $\boldsymbol{A}$. GAPDH was used as an internal control. $\boldsymbol{C}$, Western blotting analysis using spinal cord protein from wild-type $(+/+)$, heterozygous $(+/-)$, and DINE-deficient $(-/-)$ mice. Expression of DINE protein was not seen in DINE-deficient mice. $\boldsymbol{D}$, The mice on the right and the left are homozygous ( $-/-$ ) DINE-deficient and heterozygous $(+/-)$, respectively, at $0 \mathrm{~d}$ of age. DINE-deficient mice exhibited cyanosis and died immediately after birth. $\boldsymbol{E}$, Knock-out (KO) and wild-type (WT) allele-specific PCR-amplified DNA bands are indicated. $\boldsymbol{F}$, H\&E-stained lung sections were prepared from mice at $0 \mathrm{~d}$ of age (PO). $\boldsymbol{F}, \boldsymbol{G}$, The alveoli of wild-type littermates $(+/+)$ were extended $(\boldsymbol{F}$, arrows), whereas the alveoli of DINEdeficient mice $(-/-)$ were incompletely extended and tightly compressed (G). $\boldsymbol{H}, \boldsymbol{I}$, Crosssections of the diaphragm of a wild-type $(\boldsymbol{H},+/+)$ and a DINE-deficient $(\boldsymbol{I},-/-)$ littermate. $J$, The diaphragms from the DINE-deficient mice were thinner than those of wild-type and heterozygous mice. Bars indicate SD. ${ }^{*} p<0.05$. Scale bar: $100 \mu \mathrm{m}(\boldsymbol{F}, \boldsymbol{G}), 50 \mu \mathrm{m}(\boldsymbol{H}, \boldsymbol{I})$.

no survival of the homozygous pups suggested that the insertion of the loxP and neomycin-resistant gene might interfere with normal transcription or splicing, resulting in a $\mathrm{KO}$ phenotype (Schweizer et al., 1999) rather than conditional KO. We identified the existence of DINE-deficient embryos by genotyping (Fig. $1 D, E)$, and confirmed that DINE-deficient embryos did not express the protein nor its coding mRNA in the DINE-deficient embryonic spinal cord (Fig. 1B,C). Nevertheless, a small 
A
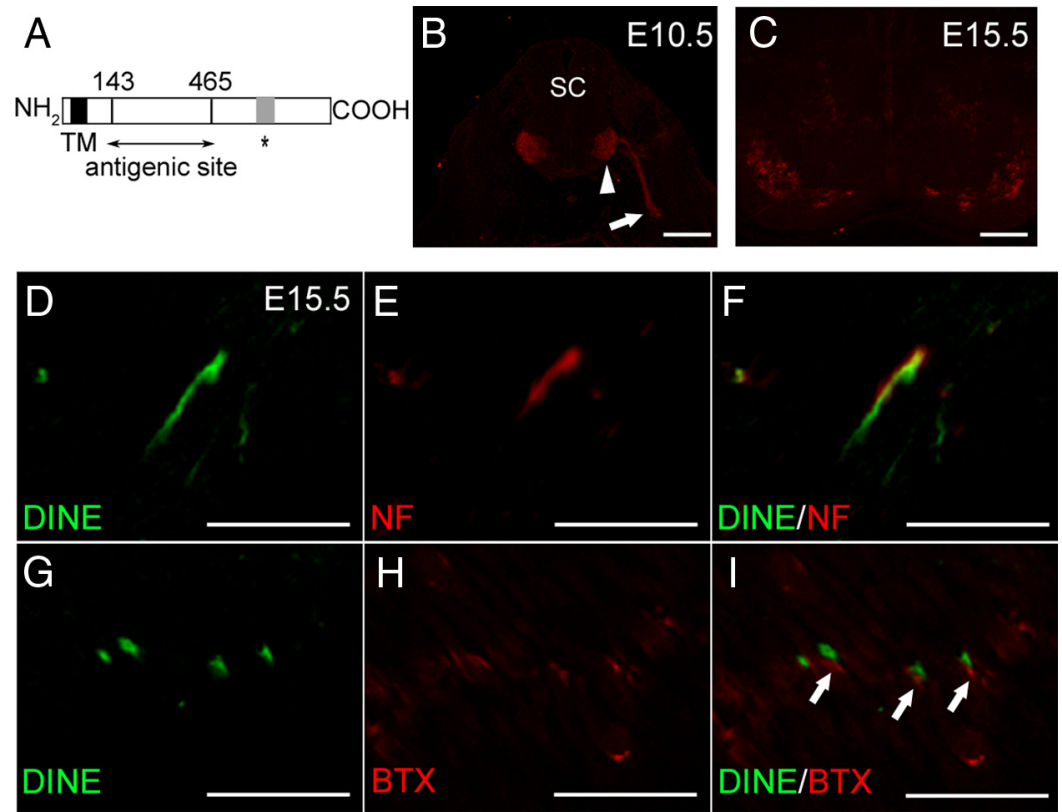

Figure 2. DINE localizes in the motor neurons and their axons from early embryonic stages. $\boldsymbol{A}$, Schematic representation of antigenic site (143-465aa) for the DINE antibody. TM and the marked region ${ }^{*}$ ) represent transmembrane region and zinc binding motif, respectively. $\boldsymbol{B}$, DINE was expressed in motor neurons (arrowhead) and their axons (arrow) at E10.5. C, DINE expression was maintained in motor neurons atE15.5. D-I, Coimmunostaining ofDINE (D, G, green) with either neurofilament (NF) ( $\boldsymbol{E}$, red) or BTX ( $\boldsymbol{H}$, red) using sections of embryonic gastrocnemius muscle. $\boldsymbol{F}$ and $\boldsymbol{I}$ indicate the merged images. DINE immunoreactivity colocalized with neurofilament $(\boldsymbol{F})$ and was attached to the postsynaptic structure (I, arrow). SC, Spinal cord. Scale bar: $100 \mu \mathrm{m}(\boldsymbol{B}, \mathbf{C}), 50 \mu \mathrm{m}(\boldsymbol{D}-\boldsymbol{I})$.

amount of long mRNA containing the neomycin-resistant gene sequence was found; presumably the mutated mRNA was degraded quickly and translation could not occur.

The DINE-deficient mice died immediately after birth (Fig. $1 D)$. Histological analysis of the newborn pups revealed that the alveoli of P0 DINE-deficient mice were tightly compressed when compared with the extended alveoli of wild-type littermates (Fig. $1 F, G)$. Further, extensive histological survey of various organs and tissues using H\&E staining demonstrated that the diaphragm thickness in DINE-deficient mice (127.7 $\pm 10.9 \mu \mathrm{m})$ was significantly reduced compared with wild-type $(147.9 \pm 12.3 \mu \mathrm{m}$, $p<0.05)$ and heterozygous $(161.6 \pm 22.2 \mu \mathrm{m}, p<0.05)$ mice (Fig. $1 H-J$ ). The diaphragm defects were assumed to contribute to the respiratory failure. DINE expression is restricted to the nervous system and is not localized in skeletal muscle (Nagata et al., 2006) (Fig. 2), suggesting that this severe atrophy of the skeletal muscle was likely caused by a failure of nerve innervation and that DINE gene disruption may affect motor neuron survival or innervation of skeletal muscle.

\section{DINE expression in axon terminal of motor neuron}

DINE mRNA was previously reported to be specifically localized in some groups of neurons from early developmental stages (Nagata et al., 2006). We examined the localization of DINE protein using a newly generated antibody (Fig. $2 A$ ). DINE was expressed in motor neurons (Fig. $2 B$, arrowhead) and their axons (Fig. $2 B$, arrow) at E10.5. Similar expression was also observed at E15.5 (Fig. 2C), with expansion into the axon terminals in the skeletal muscle (Fig. 2D-I). DINE immunostaining at E15.5 overlapped with neurofilament staining (indicating axon expression) (Fig. 2 F), and was apposed to BTX staining (indicating postsynaptic expression) (Fig. $2 I$ ). These data suggest that DINE was also expressed at the axon terminal.

\section{Numbers of motor neurons and their projections to targets are normal in DINE-deficient mice}

To examine the potential for degeneration of motor neurons in the spinal cord, we counted the number of motor neurons in the C3-C5 segments of E18.5 spinal cord using ChAT immunostaining as a motor neuron marker. There was no difference in numbers of ChAT-positive neurons in the ventral horn between the DINEdeficient and wild-type mice (Fig. 3A-C). To assess whether motor neurons projected normally to the target muscles, we examined peripheral nerve projection from the CNS using neurofilament immunohistochemistry in a whole-mount embryo preparation. There were no differences in the morphology of the peripheral nerve branching and formation of plexuses between the DINE-deficient and wild-type mice at E10.5 (Fig. 3D-G). The branching patterns of the cranial nerves, including the trigeminal, facial, and vagus nerves, and of the spinal nerves, including the nerve plexus, were normal in DINEdeficient mice. The phrenic nerve that consisted of motor axons originating in the spinal cord between C3 and C5 and projecting into the diaphragm, and its branching from the spinal cord, also appeared normal in DINEdeficient mice. These data suggest that PNS projections and formation of branching from the brain and the spinal cord were normal in DINE-deficient mice.

\section{Final branching of phrenic nerve in the diaphragm is perturbed in DINE-deficient mice}

We analyzed the precise termination of the phrenic nerves on the diaphragm at various developmental stages using neurofilament immunostaining of whole-mount diaphragms from DINEdeficient and wild-type mice. In wild-type mice, the phrenic nerve normally reached the diaphragm at E12.5 (Fig. 4A, C). The axons were branched and extended from the nerve trunks and terminated at the NMJ. Although numerous small branches from the trunk of the phrenic nerve were seen in the diaphragm of wild-type mice at this stage (Fig. 4C), such fine branches from the trunk were not observed in DINE-deficient mice (Fig. $4 B, D$ ). These reductions of the fine branches were more clearly seen in E15.5 diaphragm of DINE-deficient mice (supplemental Fig. S1, available at www.jneurosci.org as supplemental material). No obvious differences between wild-type and heterozygous mice were observed (supplemental Fig. S1, available at www.jneurosci. org as supplemental material). Further, although the tip of the phrenic nerve trunk did enter into and reach the appropriate initial position in the diaphragm in DINE-deficient mice, the final branching from the tip of the nerve trunk appeared to be disrupted. As such, the numbers of branching fibers in DINEdeficient mice was reduced to approximately one-sixth of that in wild-type mice ( $2.7 \pm 0.6$ branches in DINE-deficient embryos, $n=3$, vs $17.3 \pm 0.6$ branches in wild-type embryos, $n=3, p<$ $0.001)$. These data suggest that DINE can regulate the final branching and termination of nerves in the target muscles, but 
does not contribute to proper branching from the CNS or to nerve guidance to the target muscles.

\section{Normal AChR clustering in the postsynaptic structure of DINE-deficient mice}

To clarify the role of DINE in the formation of the postsynaptic structure at NMJ, we examined postsynaptic AChR clustering. We stained the diaphragm with antineurofilament antibody and BTX as a marker for AChR. As the postsynaptic structure was unclear at E12.5, we used diaphragms from E15.5 when the clustering formation of AChR at NMJ was clearly observed. At this stage, the phrenic nerve innervated the whole muscle in wild-type embryos (Fig. 5A,C,G), whereas only a few thick branches without the secondary and tertiary branches from the phrenic nerve trunk were observed in DINEdeficient mice (Fig. 5B,D,H). Nevertheless, AChR clustering appeared normal in DINE-deficient mice. The localization of the BTX-labeled structure, which comprised a stream of AChR clustering, was similar between DINE-deficient and wildtype mice (Fig. $5 E, F$ ). Merged images of BTX and neurofilament staining demonstrated that fine axon terminals were close to almost all AChR clusters in wild-type mice, whereas in DINE-deficient mice the primary thick phrenic nerve branch only attached to a few AChR clusters (Fig. $5 G, H)$. After E15.5, the intensity of BTX labeling in DINE-deficient mice quickly disappeared, except near the thick primary branches. We also examined mRNA expression levels of various components of the postsynaptic structure. mRNA for the MuSK and the LRP4, which together form the agrin receptor, the Dok-7, and AChR- $\alpha$ were all observed in the diaphragm of DINE-deficient mice at levels comparable to those in wild-type and heterozygous mice (supplemental Fig.

S2, available at www.jneurosci.org as supplemental material). These data suggest that postsynaptic structures were autonomously and transiently formed in DINE-deficient mice, and that DINE does not play a role in autonomous development of postsynaptic side structures until the failure of presynaptic termination occurs.

\section{Ultrastructure of the nerve terminal}

The appropriate terminal branching of motor neurons and their proper formation of NMJ are achieved by the function of at least three distinct components: the motor neuron (presynaptic element), the muscle cell (postsynaptic element), and the terminal Schwann cell (extra-synaptic element). We examined whether terminal Schwann cells were affected in DINE-deficient mice. We conducted whole-mount anti-S100 immunostaining to identify Schwann cells as well as the terminal Schwann cells. The
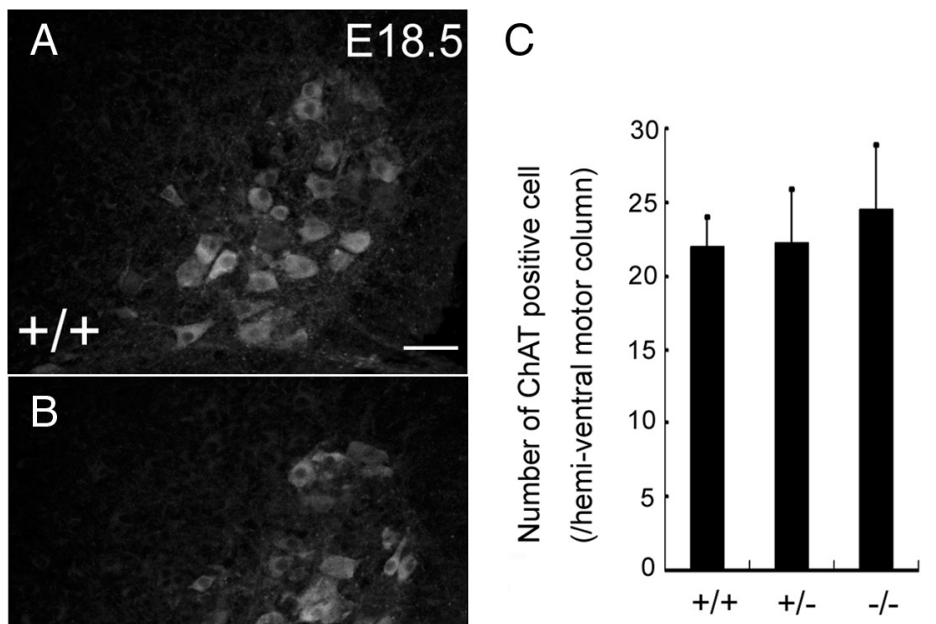

Figure 3. Number of motor neurons and their projections are normal in DINE-deficient mice. $A, B, C$ ChAT immunofluorescence demonstrating ventral motor neurons in the spinal cord $(C 3-C 5)$ at E18.5. $C$, No significant difference in numbers of ChATregion showing the presence of the phrenic nerve (Ph, arrow) adjacent to the brachial plexus (Br) in both embryos $(\boldsymbol{F}, \boldsymbol{G})$. Scale bar: $50 \mu \mathrm{m}(\boldsymbol{A}, \boldsymbol{B}), 1 \mathrm{~mm}(\boldsymbol{D}, \boldsymbol{E}), 500 \mu \mathrm{m}(\boldsymbol{F}, \boldsymbol{G})$.

S-100-positive Schwann cells along the nerve trajectory were normal in DINE-deficient mice, as were the terminal Schwann cells, which were S-100 immunoreactive and located over the BTXpositive staining (Fig. $6 A-F$ ). Ultrastructural studies also demonstrated the capping of the terminal Schwann cells over the presynaptic terminals in DINE-deficient mice (Fig. 6G,H). These data suggest that DINE deficiency does not affect the localization of terminal Schwann cells. Electron microscopy revealed that the developing nerve terminals in wild-type embryos occasionally included scattered mitochondria and synaptic vesicles, whereas nerve terminals in DINE-deficient mice showed a tendency of less mitochondria and synaptic vesicles. Although the significance of this morphological difference seen in the nerve terminal is unclear, the overall arrangement of the presynaptic terminal, muscle cell, and the terminal Schwann cell appeared normal in DINE-deficient mice. 


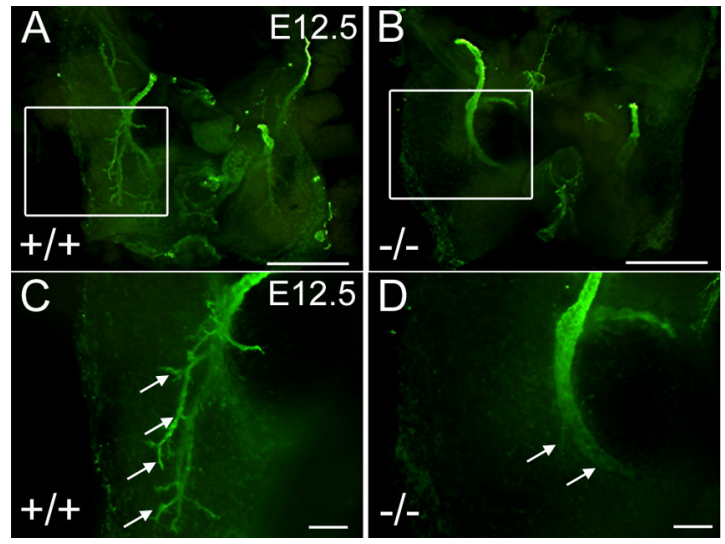

Figure 4. Aberrant innervation of the diaphragm in DINE-deficient mice. $A-D$, The nerves in the diaphragm were immunohistochemically labeled with anti-neurofilament antibodies. Cand $\boldsymbol{D}$ are high-magnification views of the frames in $\boldsymbol{A}$ and $\boldsymbol{B}$, respectively. In wild-type mice, the phrenic nerves were branched out on the diaphragm by E12.5 $(A, C)$. Many intramuscular branches were observed in wild-type mice ( $\boldsymbol{C}$, arrows), whereas only a few branches were seen in DINE-deficient mice ( $\boldsymbol{B}, \boldsymbol{D}$, arrows). Scale bar: $500 \mu \mathrm{m}(\boldsymbol{A}, \boldsymbol{B}), 100 \mu \mathrm{m}(\boldsymbol{C}, \boldsymbol{D})$.

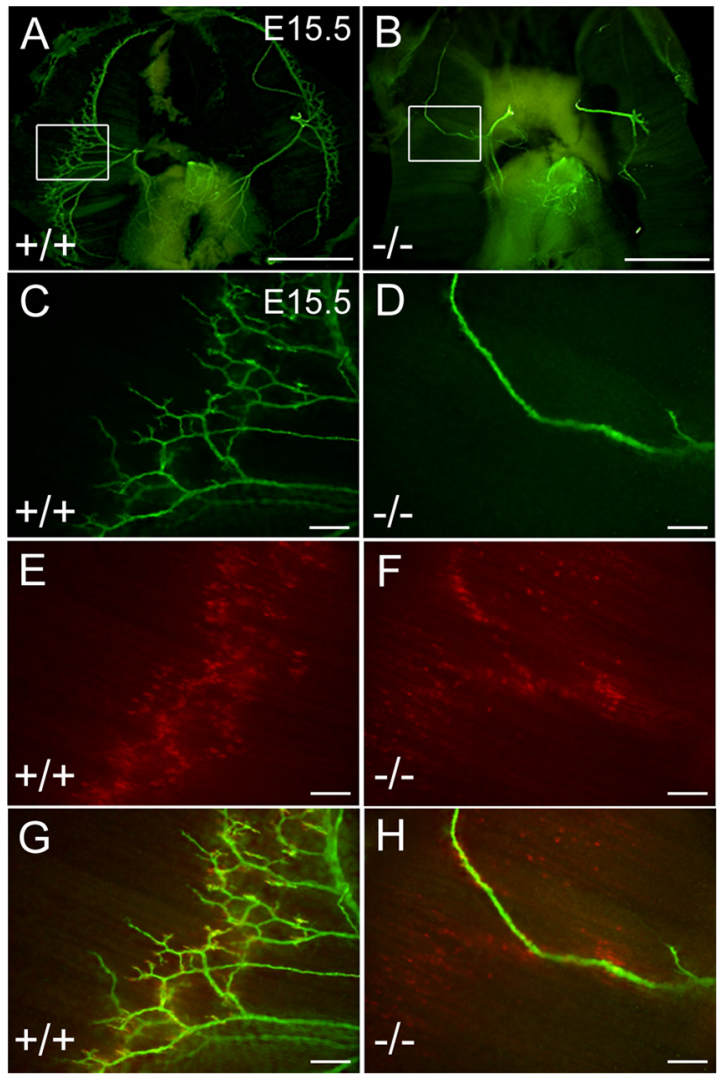

Figure 5. Diaphragms of DINE-deficient mice exhibit only a few NMJs. The diaphragms of wild-type $(\boldsymbol{A}, \boldsymbol{C}, \boldsymbol{E}, \boldsymbol{G})$ and DINE-deficient $(\boldsymbol{B}, \boldsymbol{D}, \boldsymbol{F}, \boldsymbol{H})$ mice at E15.5 were stained with antineurofilament antibodies $(\boldsymbol{A}-\boldsymbol{D})$ and with $B T X(\boldsymbol{E}, \boldsymbol{F})$. The phrenic nerves from DINE-deficient mice show complete absence of branches and only a few attached BTX-positive AChR clusters $(\boldsymbol{D}, \boldsymbol{F}), \boldsymbol{G}$ and $\boldsymbol{H}$ show the merged images of $\boldsymbol{C} / \boldsymbol{E}$ and $\boldsymbol{D} / \boldsymbol{F}$, respectively. Scale bar: $1 \mathrm{~mm}(\boldsymbol{A}, \boldsymbol{B})$, $100 \mu \mathrm{m}(\boldsymbol{C}-\boldsymbol{H})$.

\section{Innervation of other muscles is perturbed in}

\section{DINE-deficient mice}

To determine whether the failure of final branching in the target muscle occurred specifically in phrenic nerve innervation in DINE-deficient mice, we examined other muscles and their in-
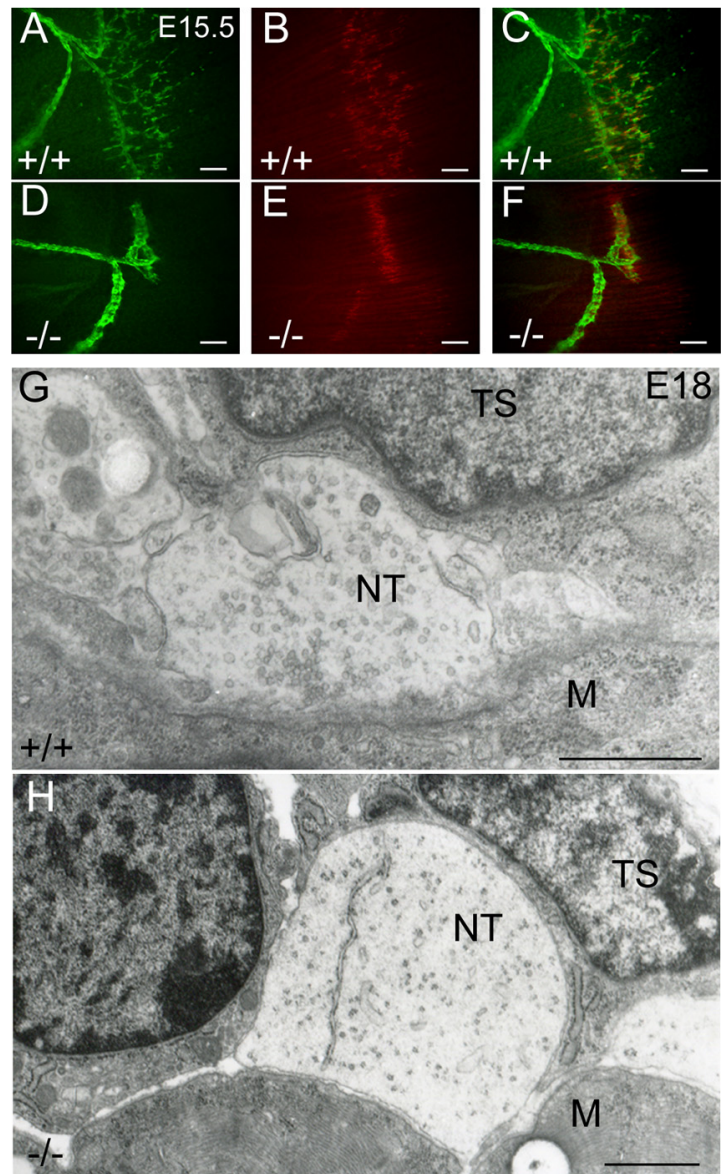

Figure 6. Localization of Schwann cells and ultrastructure of nerve terminal. $A-F$, Schwann cells, including the terminal Schwann and postsynaptic structures, were demonstrated by $S-100$ ( $\boldsymbol{A}$ and $\boldsymbol{D}$, green) and $B$ TX staining ( $\boldsymbol{B}$ and $\boldsymbol{E}$, red), respectively, in the diaphragms of wild-type $(\boldsymbol{A}-\boldsymbol{C})$ and DINE-deficient $(\boldsymbol{D}-\boldsymbol{F})$ mice. $\boldsymbol{C}$ and $\boldsymbol{F}$ demonstrate the merged images of $\boldsymbol{A} / \boldsymbol{B}$ and $\boldsymbol{D} / \boldsymbol{E}$, respectively. $\boldsymbol{G}, \boldsymbol{H}$, Ultrastructure of NMJ in wild-type $(\boldsymbol{G})$ and DINE-deficient $(\boldsymbol{H})$ mice. The nerve terminal (NT), which was attached to the surface of muscle cells (M), was capped by the terminal Schwann cells (TS) in both wild-type and DINE-deficient embryos (E18). In wild-type mice, nerve terminals occasionally had mitochondria and vesicles, whereas in DINE-deficient mice the number of mitochondria and vesicles appeared less. Scale bar: $100 \mu \mathrm{m}(\boldsymbol{A}-\boldsymbol{F}), 1 \mu \mathrm{m}(\boldsymbol{G}, \boldsymbol{H})$.

nervated nerves, including the latissimus dorsi (LD). The LD is a large muscle innervated by motor axons from the spinal cord. In wild-type mice, the thoracodorsal nerve, which projects to the LD muscle, reached the upper region of the muscle and extended into the muscle with repetitive branching (Fig. $7 A$ ). The branching nerve was finally in close proximity to the AChR clusters and made NMJs (Fig. 7 B, C). By contrast, in DINE-deficient mice, the nerve stopped branching in the upper region of the LD (Fig. 7D). This branching deficit resulted in the abolishment of AChR clusters in the $\mathrm{LD}$, except for a narrow area on the upper region (Fig. $7 E, F)$. A similar observation was found in other muscles examined, including the intercostal muscles (supplemental Fig. S3, available at www.jneurosci.org as supplemental material). These data demonstrate that the failure of final motor axon termination to the target synapse is not specific to the phrenic nerve of DINEdeficient mice. The impairment of axon terminal branching was significant in motor neurons, but similar impairment was not observed in peripheral or central terminals of the DRG neurons, at least not during embryonic development (supplemental Figs. S4 and S5, available at www.jneurosci.org as supplemental material). 


\section{Discussion}

In the present study, we demonstrated that loss of DINE perturbed the terminal branching of motor nerves in skeletal muscles. DINE-deficient mice are lethal immediately after birth, likely due to diaphragm dysfunction, which showed severe atrophy. Although there were no obvious deficits in numbers of motor neurons in the spinal cord or in nerve projections from the spinal cord to the skeletal muscle in DINE-deficient mice, a substantial decrease of nerve terminal arborization was observed in the diaphragm from E12.5, such that the diaphragms had only a few NMJs at E15.5. No clear abnormalities, including the thickness of diaphragm, motor neuron cell number, and nerve branching in diaphragm were observed in heterozygous mice. Similar changes in the nerve terminal morphology were apparent in other skeletal muscles including the LD, the intercostal muscles, and the cutaneous maximus in DINE-deficient mice. Thus, these data suggest that DINE is crucial for formation of axon arbors in the target muscles, but plays no obvious role in projection of nerves to their proper targets.

DINE is predominantly expressed in various groups of CNS and PNS neurons, particularly in all spinal and cranial motor neurons, from early embryonic stages (Nagata et al., 2006). The phenotype observed in DINE-deficient mice in the present study was particularly apparent in a subset of skeletal muscles. Intriguingly, the markedly affected muscles in DINE-deficient mice were all of a relatively large size and seemed to require numerous arbors of axon terminals in the muscles. For instance, the phrenic nerve reached the diaphragm as a thick nerve trunk by E12.5 and the nerve trunk extended toward the ventral and dorsal directions in the muscle. Along the extension of the main trunk in the diaphragm, the trunk provides the diaphragm with several long nerve arbors to establish numerous NMJs. By contrast, in the phrenic nerves in DINE-deficient mice, although the thickness and the arrival point of the phrenic nerve in the diaphragm was normal at E12.5, the nerves failed to extend long terminal branches from the main trunk. Only NMJs located adjacent to the main trunk of the phrenic nerve remained. Similarly, other relatively larger muscles, including the LD and the cutaneous maximus, showed similar phenotypes. By contrast, the thickness of muscles such as the intercostal muscle, which is innervated by very short branches from the trunk of the intercostal nerve, appeared normal in DINEdeficient mice. This is likely because the secondary and tertiary branched axons from the trunk do not necessarily elongate a longer distance toward the receptor site in the muscle, and therefore significant NMJs remains in the intercostal muscle of DINEdeficient mice, although moderate loss of NMJs was observed along the trunk of the intercostal nerve. These data suggest that DINE deficiency affected the final, longer branching of the motor nerve in the target muscle more severely, with shorter branches from the nerve trunk more likely to form NMJs at regions adjacent to the main nerve trunk. By contrast, the impairment of axon terminal branching was not seen in DRG neurons, which also expressed DINE at later embryonic stages. Although the reason for this discrepancy is unknown, it may relate to differences in the onset of DINE expression between motor and sensory neurons. DINE expression in motor neuron begins at early embryonic stages, whereas the onset of DINE expression in DRG neurons occurs at later embryonic stages (Kato et al., 2002). As such, we found no differences in central and peripheral terminal branches of sensory neurons at the embryonic stages examined. Alternatively, a muscle-derived factor may affect the DINE-mediated final branching, and thus the impairment may be seen specifically in motor axons.

A failure of NMJ formation may be caused by dysfunctioning molecules in the axon, the skeletal muscle, or the terminal Schwann cell (Lin et al., 2000; Misgeld et al., 2002, 2005; Uetani et al., 2006; Liu et al., 2008). The NMJ includes the aggregation of densely packed clusters of AChRs opposite the motor nerve terminal, which in turn requires an orchestration of molecules in both presynapses and postsynapses. The release of agrin from the axon terminal induces the aggregation of AChR in skeletal muscle, whereas in the postsynaptic side a molecular formation consisting of MuSK and LRP4 (DeChiara et al., 1996; Glass et al., 1996; Zhang et al., 2008), which together form the agrin receptor, is required. In addition, Dok-7 was identified at the postsynaptic region of the NMJ and shown to be important in forming AChR clusters and NMJs (Beeson et al., 2006). Nevertheless, the mRNA expression of those molecules was normal in the diaphragm of DINE-deficient mice, as was the integrity of NMJ formation adjacent to the nerve trunk, suggesting a normal function of those molecules. It was also reported that nascent AChR clusters are formed in a nerve-independent manner at E13.5-E16.5, whereas most neuromuscular junctions are formed in a nerve-dependent 
manner at E18.5 (Okada et al., 2006). In DINE-deficient mice, there was a transient AChR clustering in the diaphragm at E14.5E15.5, forming a continual ventrodorsal line where NMJs are normally formed. However, the loss of the fine arborization of the phrenic nerve in DINE-deficient mice causes the failure of axon contacts to muscles, resulting in the disappearance of AChR clusters after E15.5, although NMJs lying adjacent to the major trunk were maintained. These data suggest that the muscles, including their composition of molecules necessary to form and maintain NMJs, develop normally at least until nerve innervation occurs in DINE-deficient mice.

Similar branching defects were reported in mice deficient in other genes (Haase et al., 2002; Livet et al., 2002). For instance, Pea3, which belongs to the family of ETS transcription factors, is expressed in segments $\mathrm{C} 5-\mathrm{C} 8$ of the spinal cord, and those motor neurons innervate skeletal muscles in the forelimb and the shoulder girdle, including the LD and the cutaneous maximus. Loss of Pea3 function causes dramatic defects in the arborization and innervation of the target muscles of Pea3-positive motor neurons, an innervation defect similar to the phenotype seen in those two muscles in DINE-deficient mice. However, Pea3 mRNA expression in the spinal cord was normal in DINE-deficient mouse (data not shown). Further, although Pea3 was expressed by particular motor pools and with dependence of target (Haase et al., 2002), DINE was expressed by all motor neurons. Thus, the branching defect in DINE-deficient mice is unlikely associated with Pea3 gene expression.

Terminal Schwann cells have been shown to play a critical role in innervation of skeletal muscles (Lin et al., 2000). Thus, we examined whether absence of DINE affects the distribution of terminal Schwann cells. As the DINE-deficient diaphragm exhibited normal capping of nerve terminals by the terminal Schwann cells, we consider DINE to have no effect on the adhesion and distribution of these cells. By electron microscopy, scattered mitochondria and synaptic vesicles were occasionally observed in the wild-type nerve terminals, whereas the amount of these small organelles seemed less in DINE-deficient nerve terminals. At present, we are unable to determine the significance of this observation. However, this may suggest a loss of integrity or an unhealthy feature of terminals in DINE-deficient mice.

In terms of the association between formation of nerve terminal arbors and protease activity, it is possible that DINE may cleave some membrane or secreted proteins to induce the formation of final axon arbors. It was recently shown that Meltrin- $\beta$, a transmembrane-type metallopeptidase, plays a regulatory role in formation of the NMJ (Yumoto et al., 2008). Intriguingly, this function was unrelated to the enzymatic function of Meltrin- $\beta$. Instead, Meltrin- $\beta$ could bind to EphA 4 and block the internalization of the EphA4-Ephrin-A5 complexes, thus preventing receipt of a repulsion signal at the NMJ of the axon terminal and the muscle. Similarly, NEP interacts with PTEN via its intracellular domain and can inhibit the PI3K/Akt oncogenic pathway (Sumitomo et al., 2004). By analogy, DINE may also have other functions in terminal branching besides peptidase activity and this may be a reason why previous efforts were unsuccessful in identifying DINE ligands (Valdenaire et al., 1999; Shirotani et al., 2001).

In conclusion, we demonstrated that although DINE is not involved in the maintenance of motor neurons, initial axonal growth, trajectories, or invasion into muscles, loss of DINE expression perturbs the final arborization of motor axons. Axonal arborization at the target muscle is an essential event for normal development, as well as for regeneration, which may reflect the abundant expression of DINE during embryonic development and its marked induction during nerve regeneration in motor neurons, respectively. As DINE expression is also seen in some groups of neurons in the CNS, DINE deficiency may perturb axonal arbors in CNS neurons as well. Although the brains of DINE-deficient mice appeared normal, more precise examination of synapse formation is required, as well as the identification of possible DINE substrate peptides.

\section{References}

Beeson D, Higuchi O, Palace J, Cossins J, Spearman H, Maxwell S, NewsomDavis J, Burke G, Fawcett P, Motomura M, Müller JS, Lochmüller H, Slater C, Vincent A, Yamanashi Y (2006) Dok-7 mutations underlie a neuromuscular junction synaptopathy. Science 313:1975-1978.

Copeland NG, Jenkins NA, Court DL (2001) Recombineering: a powerful new tool for mouse functional genomics. Nat Rev Genet 2:769-779.

DeChiara TM, Bowen DC, Valenzuela DM, Simmons MV, Poueymirou WT, Thomas S, Kinetz E, Compton DL, Rojas E, Park JS, Smith C, DiStefano PS, Glass DJ, Burden SJ, Yancopoulos GD (1996) The receptor tyrosine kinase MuSK is required for neuromuscular junction formation in vivo. Cell 85:501-512.

Devault A, Lazure C, Nault C, Le Moual H, Seidah NG, Chrétien M, Kahn P, Powell J, Mallet J, Beaumont A (1987) Amino acid sequence of rabbit kidney neutral endopeptidase 24.11 (enkephalinase) deduced from a complementary DNA. EMBO J 6:1317-1322.

Glass DJ, Bowen DC, Stitt TN, Radziejewski C, Bruno J, Ryan TE, Gies DR, Shah S, Mattsson K, Burden SJ, DiStefano PS, Valenzuela DM, DeChiara TM, Yancopoulos GD (1996) Agrin acts via a MuSK receptor complex. Cell 85:513-523.

Haase G, Dessaud E, Garcès A, de Bovis B, Birling M, Filippi P, Schmalbruch $\mathrm{H}$, Arber S, deLapeyrière O (2002) GDNF acts through PEA3 to regulate cell body positioning and muscle innervation of specific motor neuron pools. Neuron 35:893-905.

Kato R, Kiryu-Seo S, Kiyama H (2002) Damage-induced neuronal endopeptidase (DINE/ECEL) expression is regulated by leukemia inhibitory factor and deprivation of nerve growth factor in rat sensory ganglia after nerve injury. J Neurosci 22:9410-9418.

Kiryu S, Yao GL, Morita N, Kato H, Kiyama H (1995) Nerve injury enhances rat neuronal glutamate transporter expression: identification by differential display PCR. J Neurosci 15:7872-7878.

Kiryu-Seo S, Sasaki M, Yokohama H, Nakagomi S, Hirayama T, Aoki S, Wada K, Kiyama H (2000) Damage-induced neuronal endopeptidase (DINE) is a unique metallopeptidase expressed in response to neuronal damage and activates superoxide scavengers. Proc Natl Acad Sci USA 97:4345-4350.

Kiryu-Seo S, Kato R, Ogawa T, Nakagomi S, Nagata K, Kiyama H (2008) Neuronal injury-inducible gene is synergistically regulated by ATF3, c-Jun, and STAT3 through the interaction with Sp1 in damaged neurons. J Biol Chem 283:6988-6996.

Kopp DM, Trachtenberg JT, Thompson WJ (1997) Glial growth factor rescues Schwann cells of mechanoreceptors from denervation-induced apoptosis. J Neurosci 17:6697-6706.

Lin W, Sanchez HB, Deerinck T, Morris JK, Ellisman M, Lee KF (2000) Aberrant development of motor axons and neuromuscular synapses in erbB2-deficient mice. Proc Natl Acad Sci U S A 97:1299-1304.

Lin W, Dominguez B, Yang J, Aryal P, Brandon EP, Gage FH, Lee KF (2005) Neurotransmitter acetylcholine negatively regulates neuromuscular synapse formation by a Cdk5-dependent mechanism. Neuron 46:569-579.

Liu Y, Padgett D, Takahashi M, Li H, Sayeed A, Teichert RW, Olivera BM, McArdle JJ, Green WN, Lin W (2008) Essential roles of the acetylcholine receptor gamma-subunit in neuromuscular synaptic patterning. Development 135:1957-1967.

Livet J, Sigrist M, Stroebel S, De Paola V, Price SR, Henderson CE, Jessell TM, Arber S (2002) ETS gene Pea3 controls the central position and terminal arborization of specific motor neuron pools. Neuron 35:877-892.

Misgeld T, Burgess RW, Lewis RM, Cunningham JM, Lichtman JW, Sanes JR (2002) Roles of neurotransmitter in synapse formation: development of neuromuscular junctions lacking choline acetyltransferase. Neuron 36:635-648.

Misgeld T, Kummer TT, Lichtman JW, Sanes JR (2005) Agrin promotes 
synaptic differentiation by counteracting an inhibitory effect of neurotransmitter. Proc Natl Acad Sci U S A 102:11088-11093.

Nagata K, Kiryu-Seo S, Kiyama H (2006) Localization and ontogeny of damage-induced neuronal endopeptidase mRNA-expressing neurons in the rat nervous system. Neuroscience 141:299-310.

Ohba N, Kiryu-Seo S, Maeda M, Muraoka M, Ishii M, Kiyama H (2004) Expression of damage-induced neuronal endopeptidase (DINE) mRNA in peri-infarct cortical and thalamic neurons following middle cerebral artery occlusion. J Neurochem 91:956-964.

Okada K, Inoue A, Okada M, Murata Y, Kakuta S, Jigami T, Kubo S, Shiraishi H, Eguchi K, Motomura M, Akiyama T, Iwakura Y, Higuchi O, Yamanashi Y (2006) The muscle protein Dok-7 is essential for neuromuscular synaptogenesis. Science 312:1802-1805.

Qiu Y, Pereira FA, DeMayo FJ, Lydon JP, TsaiSY, Tsai MJ (1997) Null mutation of mCOUP-TFI results in defects in morphogenesis of the glossopharyngeal ganglion, axonal projection, and arborization. Genes Dev 11:1925-1937.

Schweizer A, Valdenaire O, Köster A, Lang Y, Schmitt G, Lenz B, Bluethmann H, Rohrer J (1999) Neonatal lethality in mice deficient in XCE, a novel member of the endothelin-converting enzyme and neutral endopeptidase family. J Biol Chem 274:20450-20456.

Shirotani K, Tsubuki S, Iwata N, Takaki Y, Harigaya W, Maruyama K, KiryuSeo S, Kiyama H, Iwata H, Tomita T, Iwatsubo T, Saido TC (2001) Neprilysin degrades both amyloid beta peptides 1-40 and 1-42 most rapidly and efficiently among thiorphan- and phosphoramidon-sensitive endopeptidases. J Biol Chem 276:21895-21901.

Sumitomo M, Iwase A, Zheng R, Navarro D, Kaminetzky D, Shen R, Georgescu
MM, Nanus DM (2004) Synergy in tumor suppression by direct interaction of neutral endopeptidase with PTEN. Cancer Cell 5:67-78.

Tanabe K, Nakagomi S, Kiryu-Seo S, Namikawa K, Imai Y, Ochi T, Tohyama M, Kiyama H (1999) Expressed-sequence-tag approach to identify differentially expressed genes following peripheral nerve axotomy. Brain Res Mol Brain Res 64:34-40.

Turner AJ, Isaac RE, Coates D (2001) The neprilysin (NEP) family of zinc metalloendopeptidases: genomics and function. Bioessays 23:261-269.

Uetani N, Chagnon MJ, Kennedy TE, Iwakura Y, Tremblay ML (2006) Mammalian motoneuron axon targeting requires receptor protein tyrosine phosphatases sigma and delta. J Neurosci 26:5872-5880.

Valdenaire O, Schweizer A (2000) Endothelin-converting enzyme-like 1 (ECEL1; 'XCE'): a putative metallopeptidase crucially involved in the nervous control of respiration. Biochem Soc Trans 28:426-430.

Valdenaire O, Richards JG, Faull RL, Schweizer A (1999) XCE, a new member of the endothelin-converting enzyme and neutral endopeptidase family, is preferentially expressed in the CNS. Brain Res Mol Brain Res 64:211-221.

Xu D, Emoto N, Giaid A, Slaughter C, Kaw S, deWit D, Yanagisawa M (1994) ECE-1: a membrane-bound metalloprotease that catalyzes the proteolytic activation of big endothelin-1. Cell 78:473-485.

Yumoto N, Wakatsuki S, Kurisaki T, Hara Y, Osumi N, Frisén J, SeharaFujisawa A (2008) Meltrin beta/ADAM19 interacting with EphA4 in developing neural cells participates in formation of the neuromuscular junction. PLoS One 3:e3322.

Zhang B, Luo S, Wang Q, Suzuki T, Xiong WC, Mei L (2008) LRP4 serves as a coreceptor of agrin. Neuron 60:285-297. 